\title{
Efficacy of Combined Levonorgestrel-Releasing Intrauterine System with Gonadotropin-Releasing Hormone Analog for the Treatment of Adenomyosis
}

\author{
Peihai Zhang $^{\mathrm{a}}$ Kun Song $^{\mathrm{a}}$ Li Li $^{\mathrm{a}}$ Kazunori Yukuwab Beihua Kong ${ }^{\mathrm{a}}$ \\ a Department of Obstetrics and Gynecology, Qilu Hospital of Shandong University, Jinan, Shandong Province, PR China; \\ ${ }^{b}$ Department of Physiology, Faculty of Pharmacy, Meijo University, Meijo, Japan
}

\section{Key Words}

Adenomyosis · Gonadotropin-releasing hormone analog •

Levonorgestrel-releasing intrauterine system with LNG-IUS implantation were few. Conclusion: The findings indicate that combined GnRHa and LNG-IUS treatment was efficacious in patients with enlarged adenomyosis.

Copyright $\odot 2013$ S. Karger AG, Basel

\section{Introduction}

Adenomyosis is common in women of childbearing age. The signs and symptoms include dysmenorrhea, menorrhagia, abnormal uterine bleeding, enlarged uterus, dyspareunia, and infertility, which can seriously affect the patient's quality of life [1]. Currently, the treatments mainly include pharmacotherapy such as oral contraceptives, nonsteroidal anti-inflammatory painkillers, progesterone, gonadotropin-releasing hormone analog $(\mathrm{GnRHa})$ and surgery [2]. Previous studies have shown the contraceptive levonorgestrel-releasing intrauterine system (LNG-IUS, Mirena) to be an effective means of treating adenomyosis. Potential mechanisms of Mirena action are endometrial decidualization and atrophy, reducing endometrial blood flow, and a decrease in the number of estrogen receptors in the endometrial glands and stroma. This may further prevent estrogen stimulation of myometrial adenomyosis causing the lesions to atrophy. The subsequent improvements in uterine smooth muscle contractility and reduced menstrual flow may explain the reduction in uterine

\begin{tabular}{ll}
\hline KARGER & $\begin{array}{l}\text { ○ 2013 S. Karger AG, Basel } \\
1011-7571 / 13 / 0225-0480 \$ 38.00 / 0 \quad \text { Karger }\end{array}$ \\
E-Mail karger@karger.com & $\begin{array}{l}\text { This is an Open Access article licensed under the terms of the } \\
\text { Creative Commons Attribution-NonCommercial 3.0 Un- } \\
\text { ported license (CC BY-NC) (www.karger.com/OA-license), } \\
\text { applicable to the online version of the article only. Distribu- } \\
\text { tion permitted for non-commercial purposes only. }\end{array}$
\end{tabular}

Kun Song, $\mathrm{MD}, \mathrm{PhD}$

Department of Obstetrics and Gynecology

Qilu Hospital, Shandong University

Shandong Province (PR China)

E-Mail songkun2001226@yahoo.com.cn 
volume [3]. For adenomyosis patients with excessive menstruation, average blood loss could be reduced by $75 \%$ after the administration of Mirena [4]. However, its efficacy in the treatment of larger uterine adenomyosis is not clear, and its ability to reduce the volume of the uterus has not yet been determined. In clinical practice, an enlarged uterus (uterine volume greater than at 12 weeks gestation) is one of the main causes of Mirena expulsion [4].

The GnRHa can effectively control endometriosis and reduce uterine volume and treat dysmenorrhea. Nevertheless, the volume of the uterus and uterine fibroids increases rapidly after termination of treatment. Furthermore, long-term administration of $\mathrm{GnRHa}$ is expensive and can lead to low estrogen levels and osteoporosis. Mirena can continuously release intrauterine levonorgestrel and is suitable for long-term treatment. So, theoretically, the combination of these two therapies could not only reduce the cost of treatment but also avoid the need for GnRHa-induced low estrogen levels. In our study, GnRHa was used to reduce uterine volume followed by the placement of Mirena. We evaluated the clinical outcome of this combined therapy in adenomyosis patients with enlarged uteruses.

\section{Subjects and Methods}

\section{Patients}

This study included 21 patients, 5 adenomyosis patients with enlarged uteruses (greater than at 12 weeks' gestation) who had also experienced Mirena expulsion within 1-2 months of implantation were evaluated. Because the desired therapeutic effect was not achieved, GnRHa was administered to reduce the volume of the uterus. First, GnRHa was used to moderately reduce uterine volume $(<10 \mathrm{~cm}$ diameter) and then Mirena was reimplanted.

Of these 5 patients, 1 opted not to enroll in this study, but instead underwent a hysterectomy. The 4 remaining patients participated after giving informed consent. The remaining $17 \mathrm{pa}-$ tients, including 1 adenomyoma relapse patient, had undergone surgical excision 6 years ago. All 21 adenomyosis patients (aged 38-42) had uterine volumes greater than 12 weeks gestational size (between 12 and 16 weeks of gestation), had finished with childbearing, did not wish to undergo surgery, and showed no contraindications for the use of steroid hormones and intrauterine devices. All patients had moderate-to-severe dysmenorrhea and increased menstrual flow. None of the patients participating in the study had any other complications.

\section{GnRHa Application}

Subcutaneous injection of goserelin (Zoladex $3.6 \mathrm{mg}$, AstraZeneca) during days 1-3 of the menstrual cycle at an interval of 28 days was done. Treatment lasted through 3-4 cycles based on the degree of reduction in uterine size. None of the patients had ever undergone estrogen add-back therapy.

GnRHa-Mirena Combined Therapy for Adenomyosis

\section{Mirena Implantation}

When uterine length was determined to be $\leq 10 \mathrm{~cm}$ through ultrasound, Mirena (Bayer, Germany) was implanted by a specialist (P.Z.) in strict accordance with the operating instructions. Positioning was then determined through combined transvaginal and transabdominal ultrasound 1 week after implantation.

\section{Outcome Measures}

Pretreatment for uterine volume and longitudinal diameter were measured five times: prior to $\mathrm{GnRHa}$ treatment, prior to $\mathrm{Mi}$ rena implantation, and at 3, 6 and 12 months after implantation. Uterine volume was calculated using the following formula: $0.523 \times a \times b \times c$. The variables $a, b$, and $c$ represent the uterine $3 \mathrm{D}$ warp values. Uterine length and volume only were chosen for the evaluation of the therapeutic effect because other adenomyosis ultrasound signs, such as subendometrial microcysts and heterogeneous myometrium appearance are not sufficiently reproducible for pre- and posttherapeutic analysis.

The degree of dysmenorrhea (pain) was assessed using the visual analogue scale. The left end of the scale was set at 0 (no pain) and the right end of scale at 100 (intolerable pain). The degree of pain was self-reported by patients.

For menstrual flow, the number of sanitary napkins used was counted. For baseline (100\%), it was the number used before treatment. The posttreatment values are given as a percentage of the pretreatment values.

Other outcome measures included LNG-IUS expulsion rate, menstrual recovery time, irregular vaginal bleeding, weight gain, nipple pain, and other related symptoms were used. Follow-up was performed at 3, 6 and 12 months after LNG-IUS implantation to document the clinical values as described previously.

Statistical Analysis

The SPSS 13.0 software package was used for statistical analysis. $\mathrm{p}<0.05$ was considered statistically significant.

\section{Results}

Of the 21 patients, $8(38 \%)$ received implantation after three GnRHa injections and the remaining 13 (62\%) patients received implantation after four GnRHa injections. After the second injection, all patients experienced amenorrhea. Dysmenorrhea gradually disappeared. $\mathrm{Pa}-$ tients experienced symptoms of low estrogen (perimenopausal symptoms), but none underwent add-back therapy. After 3-4 cycles of administration, uterine volume decreased from $311.4 \pm 32.3$ to $220.6 \pm 17.2 \mathrm{~cm}^{3}$ and uterine length decreased from $13.2 \pm 0.8$ to $8.8 \pm 2.3 \mathrm{~cm}$, which is suitable for Mirena implantation.

After LNG-IUS implantation, menstruation was restored after $73.4 \pm 5.6$ days. In $3(14.3 \%)$ patients, the first menstrual period was heavy and approached pretreatment levels. This was controlled with the administration of hemostatic agents and oxytocin. Irregular vaginal bleeding was documented in 15 (71.4\%) patients within 


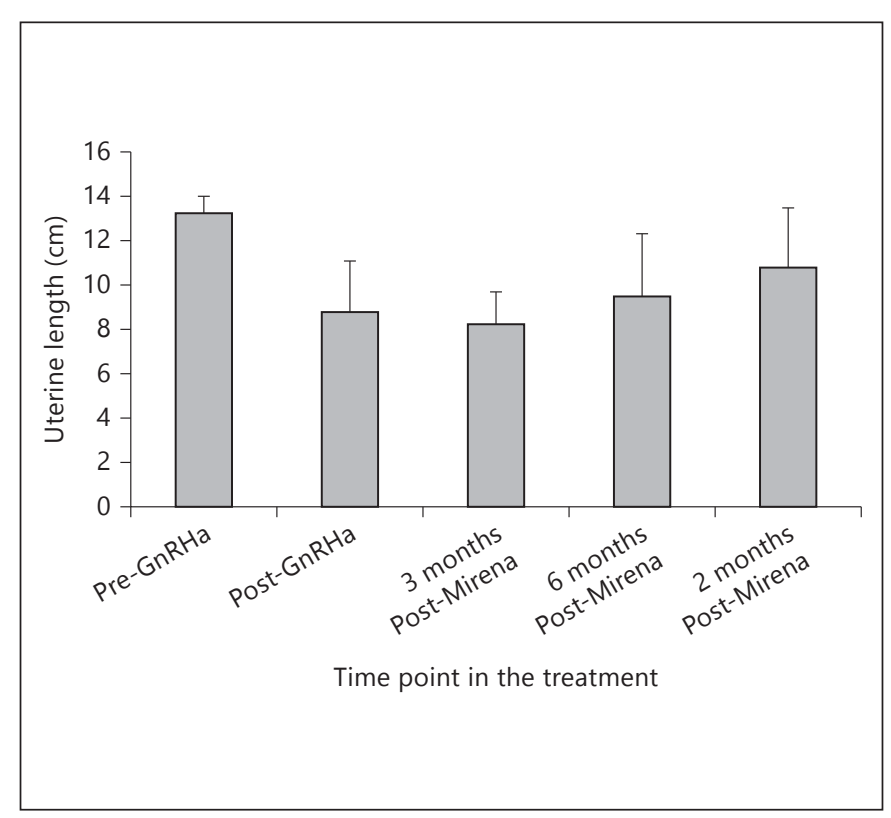

Fig. 1. Uterine length changes during the treatment.

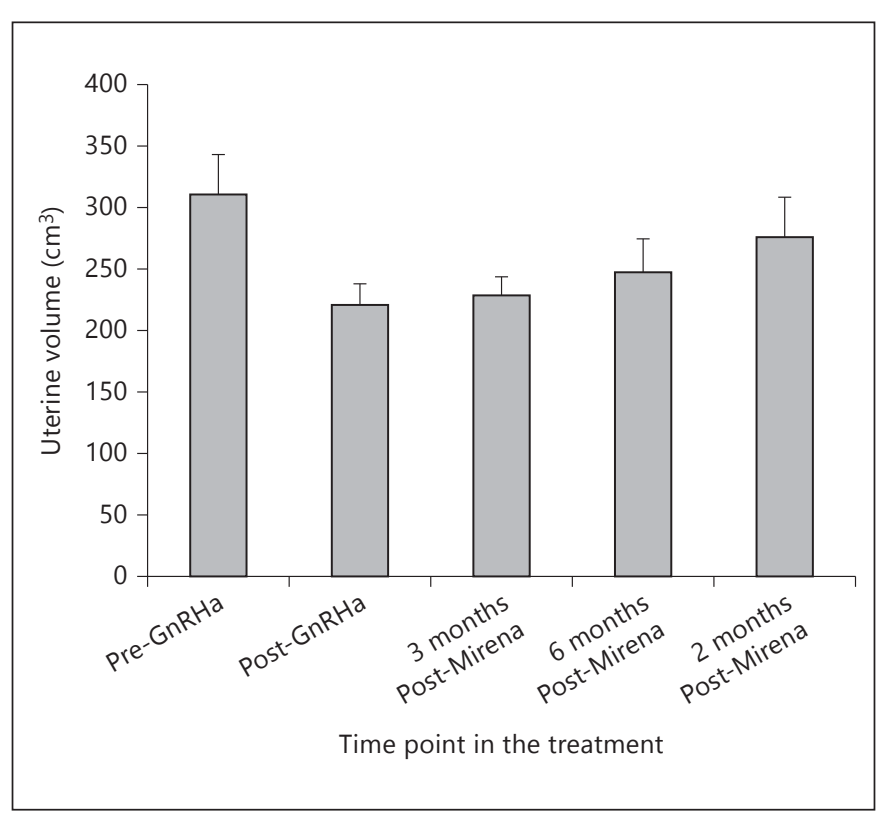

Fig. 2. Uterine volume changes during the treatment.

Table 1. Change in menstrual cycle characteristics after Mirena administration

\begin{tabular}{llllll}
\hline Time & $\mathrm{n}$ & Menstrual flow $\%$ & $\mathrm{p}^{*}$ & $\begin{array}{l}\text { Dysmenorrhea } \\
(\text { VAS) }\end{array}$ & $\mathrm{p}^{*}$ \\
\hline Pre-GnRHa & 21 & 100 & & $93.7 \pm 0.2$ & $<0.005$ \\
3 months after Mirena & 21 & $23.2 \pm 9.6$ & $<0.005$ & $21.6 \pm 5.8$ & $<0.01$ \\
6 months after Mirena & 19 & $48.6 \pm 13.7$ & $<0.01$ & $36.7 \pm 9.7$ & $<0.01$ \\
12 months after Mirena & 18 & $53.8 \pm 11.7$ & $<0.05$ & $58.2 \pm 11.5$ & \\
\hline \multicolumn{7}{l}{ Menstrual flows at 3, 6 and 12 months were calculated as percentage of pretreatment. } \\
* p value: compared with pre-GnRHa treatment. VAS = Visual analog scale. \\
\hline
\end{tabular}

6 months of Mirena implantation. Bleeding partially resolved after the administration of oral hemostatic agents. LNG-IUS was expelled in 2 patients 4 months after implantation and in 1 patient after 8 months. Dysmenorrhea recurred in these 3 patients. In patients who retained the implant $(18 / 21,85.7 \%)$, menstrual flow and dysmenorrhea were significantly lower than baseline values at 3, 6 and 12 months after implantation (table 1). Twelve months after implantation, the menstrual flow was significantly lower than baseline values $(53.8 \pm 11.7$ vs. $100, \mathrm{p}=0.03$ ). The degree of dysmenorrhea (pain) was also relieved 12 months after implantation (58.2 \pm 11.5 vs. $93.7 \pm 0.2, \mathrm{p}=0.005)$. At 3,6 and 12 months after Mirena implantation, the uterine sizes were all significantly smaller than at pre-GnRHa levels. However, uterine volume was increased at 6 and 12 months relative to postGNRHa levels. Even so, both uterine length $(10.8 \pm 2.7$ vs. $13.2 \pm 0.8, \mathrm{p}=0.04)$ and uterine volume $(276.6 \pm 32.1$ vs. $311.4 \pm 32.3, \mathrm{p}=0.005)$ were below the pre-GnRHa levels 12 months after implantation (figs. 1,2 ).

Irregular vaginal bleeding was documented in 15 (91.4\%) patients 6 months after Mirena implantation and was treated with uterotonics and Transmin. Nipple pain was documented in $2(9.5 \%)$ patients. LNG-IUS was expelled from 2 (9.5\%) patients at 4 months and from 1 patient at 8 months (expulsion rate of $14.3 \%$ or $3 / 21$ ). Side effects of Mirena implantation were well tolerated in this study. 


\section{Discussion}

This study confirmed that combined GnRHa (Mirena) and LNG-IUS protocol was an effective treatment option for an adenomyosis patient with an enlarged uterus. GnRHa reduced uterine volume effectively. Follow-up at 3, 6 and 12 months after Mirena implantation confirmed that uterine volume was reduced relative to pre-GnRHa treatment values. However, the volume of the uterus was higher at 6 and 12 months after implantation when compared with the volume at implantation. The uterine volume at 6 months was not different from the volume at 12 months. These findings suggest that Mirena was able to maintain the inhibitory state of the uterus after GnRHa treatment and effectively controlled adenomyosis. This outcome is similar to that of a previous study in which the administration of Mirena reduced blood loss $[5,6]$, uterine volume and endometrial thickness [5]. However, in another study [6], it was shown that 4 months after single GnRHa withdrawal, the uterine volume had returned to the size at baseline. Hence, a combined GnRHa-Mirena protocol was more effective in uterine volume control than single GnRHa application.

In our study, the patients' uterine volumes were so big that Mirena could be expelled without GnRHa pretreatment. After GnRHa treatment, an expulsion rate of $14.3 \%$ of IUDs could be acceptable in patients with large adenomyosis.

Surgical treatment for adenomyosis includes uterine excision, uterine artery embolization, endometrial resection, and, as the most radical approach, hysterectomy. Many young women do not like to receive surgical treat- ment based on fertility- sparing considerations. In this study, the combined GnRHa-Mirena protocol proved to be a safe and effective option for women with larger uterine adenomyosis who hope to preserve their fertility. Adenomyosis is considered to be one of the high-risk factors of infertility and accounts for 1-14\% of cases of infertility [6]. Once young patients have finished childbearing, they might opt for surgical treatment.

The combined GnRHa and Mirena protocol also costs less than surgery in China. For example, Mirena administration following 3 cycles of GnRHa would cost the equivalent of USD 6,000, while surgery such as hysterectomy would cost double the amount because of the hospital stay.

The limitations of this study include the small number of patients, not using a case-controlled trial, and the short follow-up period.

\section{Conclusion}

Our findings indicate that combined GnRHa and Mirena treatment was efficacious in patients with enlarged adenomyosis. These treatments reduced the IUD expulsion rate, relieved dysmenorrhea, reduced menstrual flow, allowed patients to avoid surgical treatment, and showed a significant clinical value.

\section{Acknowledgement}

This work was supported by grants from the National Natural Science Foundation of China (No. 81172488) to Kun Song.

\section{References}

1 Fedele L, Bianchi S, Raffaelli R, et al: Treatment of adenomyosis-associated menorrhagia with a levonorgestrel-releasing intrauterine device. Fertil Steril 1997;68:426-429.

$>2$ Fraser IS: Added health benefits of the levonorgestrel contraceptive intrauterine system and other hormonal contraceptive delivery systems. Contraception 2013;87:273-279.
-3 Fong Y, Singh K: Medical treatment of a grossly enlarged adenomyotic uterus with the levonorgestrel-releasing intrauterine system. Contraception 1999;60:173-175.

-4 Otero-Flores JB, Guerrero-Carreno FJ, Vazquez-Estrada LA: A comparative randomized study of three different IUDs in nulliparous Mexican women. Contraception 2003;67: 273-276.

5 Vercellini P, Frontino G, De Giorgi O, et al: Comparison of a levonorgestrel-releasing intrauterine device versus expectant management after conservative surgery for symptomatic endometriosis: a pilot study. Fertil Steril 2003;80:305-309.
6 Gerris J, Degueldre M, Peters AA, et al: The place of Zoladex in deferred surgery for uterine fibroids. Zoladex Myoma Study Group. Horm Res 1996;45:279-284.

7 Levy G, Dehaene A, Laurent N, et al: An update on adenomyosis. Diagn Interv Imaging 2013;94:3-25. 